\title{
Statistical Model for the Circuit Bandwidth Dependence of Low-Frequency Noise in Deep-Submicrometer MOSFETs
}

\author{
Gilson I. Wirth, Member, IEEE, Roberto da Silva, and Ralf Brederlow, Senior Member, IEEE
}

\begin{abstract}
This paper covers measurement, analytical analysis, and Monte Carlo simulation of the frequency and bandwidth dependence of MOSFET low-frequency (LF) noise behavior. The model is based on microscopic device physics parameters, which cause statistical variation in the LF noise behavior of individual devices. Analytical equations for the statistical parameters are provided. The analytical model is compared to experimental data and Monte Carlo simulation results.
\end{abstract}

Index Terms-Analog circuits, low-frequency (LF) noise, MOS transistors, noise modeling, RF circuits, variability.

\section{NOMENCLATURE}

$A_{i} \quad$ Amplitude coefficient of the Lorentzian power spectrum of the $i$ th trap.

$\left\langle A^{2}\right\rangle \quad$ Mean value of the squared amplitude coefficients $A_{i}$.

$\left\langle A^{4}\right\rangle \quad$ Mean value of $A_{i}^{4}$.

$f_{i} \quad$ Corner frequency of the Lorentzian power spectrum of the $i$ th trap.

$f_{L}, f_{H} \quad$ Lower and upper boundaries of the bandwidth of interest in a given circuit design, respectively.

$f_{\min }, f_{\max } \quad$ Lower and upper boundaries delimiting the frequency window in which random telegraph signal (RTS) is the origin of the low-frequency (LF) noise, respectively.

$L \quad$ Device channel length.

$N_{\text {dec }} \ln 10$ Trap density per unit area and frequency decade.

$N_{\text {tr }} \quad$ Actual number of traps in a particular device.

$N=\left\langle N_{\text {tr }}\right\rangle \quad$ Average number of traps in an ensemble of devices, with corner frequencies $f_{i}$ lying between $f_{\min }$ and $f_{\max }$. $n p_{\mathrm{BW}} \quad$ Noise power integrated over the bandwidth of interest in a given circuit design.

$S(f) \quad$ Noise power spectral density at frequency $f$.

$W \quad$ Device channel width.

$\sigma_{\mathrm{np}} \quad$ Standard deviation of $n p_{\mathrm{BW}}$.

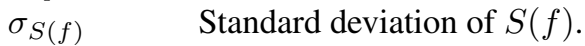

\section{INTRODUCTION}

$\mathbf{P}$ REVIOUS works show that the low-frequency (LF) noise performance of modern small-area MOS devices is dominated by random telegraph signal (RTS) fluctuations [1]-[8]. Their origin is the capture and subsequent emission of charge carriers at discrete trap levels near the $\mathrm{Si}-\mathrm{SiO}_{2}$ interface [3]-[9]. For deep-submicrometer devices, the number of traps with energy within a few $k T$ close to the surface Fermi level is small [8]. As both the number of traps and their position over the channel are random variables, noise performance may strongly vary between different devices on one chip and, moreover, even between different operation points of a single device [2], [8].

In a previous work by the same authors, an analytical modeling approach, which is based on microscopic instead of distributed quantities, was presented [2]. This paper advances statistical modeling by including Monte Carlo simulation, experimental data, and detailed modeling of noise behavior dependence on circuit bandwidth. Experimental data and Monte Carlo simulations for the dependence of statistical noise behavior on frequency are also presented with comparison to analytical model equations.

This paper is organized as follows: In Section II, the analytical statistical modeling approach presented in [2] is extended to include the frequency dependence of statistical noise behavior. In Section III, Monte Carlo simulations that corroborate the results of the analytical analysis are presented, and the model is compared to experimental data from three different technology nodes. Finally, in Section IV, the paper is concluded.

\section{Microscopic Statistical LF Noise Modeling}

Traps located in the gate oxide near the interface to the silicon capture and reemit some of the carriers responsible for the current flowing between the source and the drain of the device [3]-[10]. The resulting LF noise power spectrum may 
be written as the summation of the contribution of each one of the $N_{\text {tr }}$ traps found in the device [2], [11], i.e.,

$$
S(f)=\sum_{i=1}^{N_{\mathrm{tr}}} A_{i}^{2} \frac{1}{f_{i}} \frac{1}{1+\left(\frac{f}{f_{i}}\right)^{2}} .
$$

The Lorentzian spectrum of each trap corresponds to the Fourier transform of the autocovariance of the time-domain RTS [12]. The parameter $f_{i}$ defines the corner frequency of the Lorentzian spectrum of the $i$ th trap. Please note that $A_{i}$ is the amplitude coefficient of the Lorentzian power spectrum of the $i$ th trap (in frequency domain) and not the amplitude of the voltage $\delta V_{G}$ or current $\delta I_{D}$ fluctuation of the time-domain RTS. $A_{i}$ is related to the time-domain RTS parameters amplitude and $\beta=\tau_{c} / \tau_{e}$, where $\tau_{c}$ is the electron capture, and $\tau_{e}$ is the emission time constant, as discussed in [2].

$N=\left\langle N_{\mathrm{tr}}\right\rangle$, the average number of traps with corner frequency $f_{i}$ lying between the frequencies $f_{\min }$ and $f_{\max }$, which delimit the frequency interval in which RTS is the origin of the LF noise, is then proportional to the active device area $W \times L$ and equal to [2], [8]

$$
N=N_{\text {dec }} \ln \left(\frac{f_{\max }}{f_{\min }}\right) W L
$$

where $N_{\text {dec }} \ln 10$ is the trap density per unit area and frequency decade.

The LF noise is modeled as the superposition of individual Lorentz spectra that sum up to yield device noise behavior as given by (1).

The average value of the noise power spectral density $\langle S(f)\rangle$ evaluated by calculating the average value of (1) over $A_{i}, f_{i}$, and $N_{\mathrm{tr}}$, is [2]

$$
\langle S(f)\rangle=\frac{\left\langle A^{2}\right\rangle N_{\mathrm{dec}} W L}{f} \frac{\pi}{2}
$$

where $\left\langle A_{i}^{2}\right\rangle=\left\langle A^{2}\right\rangle$ is the average of the squared amplitude coefficients. This equation shows the commonly known $1 / f$ behavior. The details of the derivation are found in [11].

Evaluation of the standard deviation of $S(f)$ leads to [2]

$$
\sigma_{S(f)}^{2}=\frac{\left\langle A^{4}\right\rangle N_{\mathrm{dec}} W L}{2} \frac{1}{f^{2}}
$$

where $\left\langle A_{i}^{4}\right\rangle=\left\langle A^{4}\right\rangle$. The normalized standard deviation then amounts to

$$
\frac{\sigma_{S(f)}}{\langle S(f)\rangle}=\frac{\sqrt{2}}{\pi \sqrt{N_{\mathrm{dec}} W L}} \sqrt{\frac{\left\langle A^{4}\right\rangle}{\left\langle A^{2}\right\rangle^{2}}} .
$$

Here, the contributions due to scattering of the parameters $A_{i}, N_{\mathrm{tr}}$, and $f_{i}$ are all taken into account.

As can be seen in (5), the normalized standard deviation of the LF noise power spectral density $\sigma_{S(f)} /\langle S(f)\rangle$ depends on variations in the amplitude coefficients $A_{i}$ of individual traps, as given by $\left(\left\langle A^{4}\right\rangle /\left\langle A^{2}\right\rangle^{2}\right)^{0.5}$, and on the trap density and device geometry after $\left(N_{\mathrm{dec}} W L\right)^{-0.5}$. The normalized standard deviation does not depend on frequency $f$. The dependence on device geometry after $\left(N_{\mathrm{dec}} W L\right)^{-0.5}$ is a property of the Poisson statistics. $N_{\text {tr }}$ is assumed to be Poisson distributed [2]. The $\left(\left\langle A^{4}\right\rangle /\left\langle A^{2}\right\rangle^{2}\right)^{0.5}$ dependence shows that variations on the amplitude coefficients $A_{i}$ of the contribution of a trap are relevant for statistical variations in the LF noise behavior. Furthermore, since the amplitude coefficients $A_{i}$ may show strong bias point dependence, the standard deviation of the LF noise power may depend on bias point, as discussed in [2].

Earlier in this paper, the modeling of noise amplitude at a given frequency $f$ and its standard deviation were presented. However, often, the noise power integrated over the circuit bandwidth $n p_{\mathrm{BW}}$ and its related standard deviation are of even higher interest to the circuit designer. This parameter is given by the integration of (1) from $f_{L}$ to $f_{H}$, which are the lower and upper boundaries of the bandwidth of interest in a given circuit design, as follows:

$$
n p_{\mathrm{BW}}=\int_{f_{L}}^{f_{H}} S(f) d f .
$$

Inserting (1) in (6) leads to

$$
n p_{\mathrm{BW}}=\int_{f_{L}}^{f_{H}}\left(\sum_{i=1}^{N_{\mathrm{tr}}} A_{i}^{2} \frac{1}{f_{i}} \frac{1}{1+\left(\frac{f}{f_{i}}\right)^{2}}\right) d f
$$

and finally [2], [11]

$$
\left\langle n p_{\mathrm{BW}}\right\rangle=\left\langle A^{2}\right\rangle \frac{\pi}{2} \ln \left(\frac{f_{H}}{f_{L}}\right) N_{\mathrm{dec}} W L .
$$

The normalized standard deviation of the noise power spectral density in the frequency band between $f_{L}$ and $f_{H}$ is evaluated as being [2], [11]

$$
\begin{aligned}
\frac{\sigma_{n p}}{\left\langle n p_{\mathrm{BW}}\right\rangle}= & \frac{\sqrt{\left\langle n p_{\mathrm{BW}}^{2}\right\rangle-\left\langle n p_{\mathrm{BW}}\right\rangle^{2}}}{\left\langle n p_{\mathrm{BW}}\right\rangle} \\
= & \frac{2}{\pi \sqrt{N_{\mathrm{dec}} W L}} \sqrt{\frac{\left\langle A^{4}\right\rangle}{\left\langle A^{2}\right\rangle^{2}}} \\
& \times \sqrt{\frac{\int_{f_{L}}^{f_{H}} \int_{f_{L}}^{f_{H}} \frac{1}{\left(f^{\prime 2}-f^{2}\right)} \ln \left(\frac{f^{\prime}}{f}\right) d f d f^{\prime}}{\ln ^{2} \frac{f_{H}}{f_{L}}}} .
\end{aligned}
$$

The integral in the aforementioned equation has no known analytical solution. It is important to note that the last square root in the aforementioned equation, which contains the integral with no analytical solution, contains only terms in frequency $f$. Hence, the bandwidth dependence is described by the terms in this square root, as will be discussed later in this paper.

In [2], a simplification was introduced to allow the analytical evaluation of $\left\langle n p_{\mathrm{BW}}^{2}\right\rangle$, leading to an approximation for the standard deviation of the noise power in the bandwidth of interest [2, eq. (67)]. The simplification was to convert the average of a product into a product of averages in [2, eq. (63)]. In this paper, this simplification is removed, and the dependence of noise behavior on circuit bandwidth is discussed in detail. 


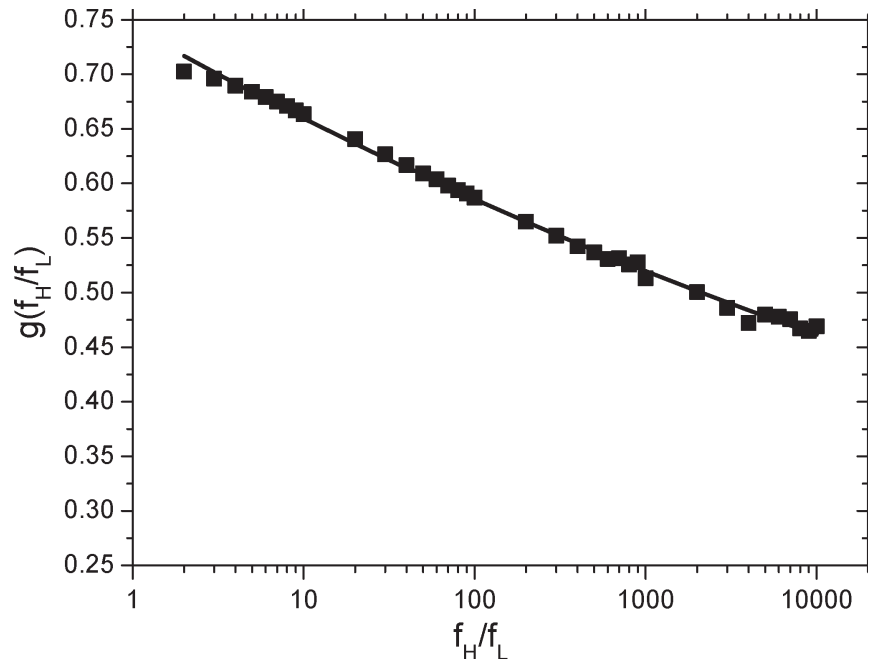

Fig. 1. Fitting function $g\left(f_{H} / f_{L}\right)$ for the integral with no analytical solution in (9). The squares are the results from the numerical integration of the term with no analytical solution in the last square root on the right-hand side of (9). The full line is the fitting function $g\left(f_{H} / f_{L}\right)$ for this term (10). A good agreement between numerical integration and fitting function is found.

The first important observation is that the integral in the square root of (9) does not depend on the absolute values of $f_{L}$ and $f_{H}$, but only on the ratio $f_{H} / f_{L}$. Furthermore, this is the only term in (9) that contains dependence on frequency. Therefore, a single variable $f_{H} / f_{L}$ can be introduced to describe the bandwidth dependence. Starting from this observation, curve-fitting techniques may be applied in order to find an appropriate fit for (9) [11]. Hence, we have

$$
\begin{aligned}
\frac{\sigma_{\mathrm{np}}}{\left\langle n p_{\mathrm{BW}}\right\rangle} & =\frac{2}{\pi} \frac{1}{\sqrt{N_{\mathrm{dec}} W L}} \sqrt{\frac{\left\langle A^{4}\right\rangle}{\left\langle A^{2}\right\rangle^{2}}} g\left(\frac{f_{H}}{f_{L}}\right) \\
& \approx \frac{2}{\pi} \frac{1}{\sqrt{N_{\mathrm{dec}} W L}} \sqrt{\frac{\left\langle A^{4}\right\rangle}{\left\langle A^{2}\right\rangle^{2}}} \frac{b}{\left(\frac{f_{H}}{f_{L}}\right)^{c}} .
\end{aligned}
$$

Here, the fitting function $g\left(f_{H} / f_{L}\right)=b /\left(f_{H} / f_{L}\right)^{c}$, where $b=0.74$ and $c=0.05$, describes the dependence on circuit bandwidth. It is a simple power law. This fitting is computationally efficient and suitable for modeling and simulation purposes in integrated circuit design. This power law fit has a fit error of order $10^{-3}$, compared to a fit error of order $10^{-4}$ for the Boltzmann fit, as discussed in [11]. However, the power law is simpler than the Boltzmann fit, and its accuracy is appropriate for modeling and circuit simulation purposes. Fig. 1 compares the numerical solution of the term with no analytical solution in (9) with the fitting equation $g\left(f_{H} / f_{L}\right)$. A very good agreement between the numerical solution and the analytical fitting equation is found.

\section{Monte Carlo Simulations And EXPERIMENTAL RESULTS}

In order to explore the LF noise behavior of small-area devices and validate the analytical equations derived in Section II, a comparison of model equations to Monte Carlo simulations and to experimental data is done in this section. The normalized standard deviation of the noise power $\sigma_{S(f)} /\langle S(f)\rangle$ depends on $A_{i}, N_{\mathrm{dec}}$, and the transistor area $W L$. The normalized standard deviation of the integrated noise power $\sigma_{\mathrm{np}} /\left\langle n p_{\mathrm{BW}}\right\rangle$ depends on $A_{i}, N_{\mathrm{dec}}$, the transistor area $W L$, and the frequency bandwidth defined by $f_{H}$ and $f_{L}$. The dependence of both $\sigma_{S(f)} /\langle S(f)\rangle$ and $\sigma_{\mathrm{np}} /\left\langle n p_{\mathrm{BW}}\right\rangle$ on $N_{\mathrm{dec}}$ and the transistor area $W L$ has been studied in previous works [2], [11]. In this paper, it is verified by experimental results and Monte Carlo simulation that $\sigma_{S(f)} /\langle S(f)\rangle$ does not depend on frequency $f$. The dependence of $\sigma_{\mathrm{np}} /\left\langle n p_{\mathrm{BW}}\right\rangle$ on the frequency bandwidth defined by $f_{H}$ and $f_{L}$ is also verified by experimental results and Monte Carlo simulation. Furthermore, the dependence of $\sigma_{S(f)} /\langle S(f)\rangle$ and $\sigma_{\mathrm{np}} /\left\langle n p_{\mathrm{BW}}\right\rangle$ on $A_{i}$ is verified by Monte Carlo simulation.

The LF noise behavior of three different CMOS technologies was experimentally characterized. The minimum transistor sizes for the $0.25-\mu \mathrm{m}$ node are $W=0.30 \mu \mathrm{m}$ and $L=0.25 \mu \mathrm{m}$ $\left(t_{\mathrm{ox}}=5 \mathrm{~nm}\right.$, nominal supply voltage $\left.V_{\mathrm{dd}}=2.5 \mathrm{~V}\right)$. For the $0.13-\mu \mathrm{m}$ node, the minimum sizes are $W=0.16 \mu \mathrm{m}$ and $L=$ $0.13 \mu \mathrm{m}\left(t_{\mathrm{ox}}=2.2 \mathrm{~nm}, V_{\mathrm{dd}}=1.5 \mathrm{~V}\right)$, and for the $0.09-\mu \mathrm{m}$ node, the minimum sizes are $W=0.12 \mu \mathrm{m}$ and $L=0.09 \mu \mathrm{m}$ $\left(t_{\mathrm{ox}}=1.6 \mathrm{~nm}, V_{\mathrm{dd}}=1.2 \mathrm{~V}\right)$. For the experimental results shown here, a total of 127 transistors were measured in the $0.13-\mu \mathrm{m}$ technology node. For the $0.25-$ and $0.09-\mu \mathrm{m}$ technology nodes, a total of 30 and 18 transistors were experimentally characterized, respectively. The details of the measurement setup are described in [13].

For model parameter extraction, first, the average number of traps per device in the measurement frequency range $\left\langle N_{\text {tr }}\right\rangle$ is extracted, as described in [1] and [2]. For the 0.25-, 0.13-, and $0.09-\mu \mathrm{m}$ technology nodes, $\left\langle N_{\mathrm{tr}}\right\rangle$ is $9.0,2.7$, and 1.8 , respectively. The measurement frequency range for the evaluation of $\left\langle N_{\text {tr }}\right\rangle$ is $1 \mathrm{~Hz}$ to $10 \mathrm{kHz}$.

After the extraction of $N=\left\langle N_{\mathrm{tr}}\right\rangle,\left(N_{\mathrm{dec}} W L\right)^{0.5}$ is calculated using (2). The term $(2 / \pi) \cdot\left(\left\langle A^{4}\right\rangle /\left\langle A^{2}\right\rangle^{2}\right)^{0.5}$ is then the only unknown term in (5) and (9) and is evaluated to bring model and experimental data into agreement. For the minimumsize transistors in the $0.25-, 0.13-$, and $0.09-\mu \mathrm{m}$ technology nodes, the term $\left(N_{\mathrm{dec}} W L\right)^{-0.5}$ is found to be equal to 1.01 , 1.85 , and 2.26, respectively. The term $N_{\mathrm{dec}} W L \ln 10$ is then the average number of number of traps per frequency decade in the device active area. The average number of traps per device in the measurement frequency range is then given by $\left\langle N_{\text {tr }}\right\rangle=N_{\text {dec }} W L \ln \left(10^{4}\right)$.

Monte Carlo simulation of the LF noise behavior of smallarea MOSFETs as the superposition of different RTS, as given by (1), is performed using the following algorithm.

1) First, an array of 268 frequency values equally spaced in a log scale is generated. Each frequency value is indexed with an index numbered from 1 to 268 . The value of $f_{\min }$ is chosen to be $1 / 1024 \mathrm{~Hz}$, and $f_{\max }$ is $10.9 \mathrm{MHz}$. The ratio of the geometric progression is $2^{(1 / 8)}$.

2) Simulations with different average values for number of traps $\left\langle N_{\text {tr }}\right\rangle$ are carried out.

3) For each sample, the number of traps in that particular sample is chosen by lot according to a Poisson distribution with an average equal to $\left\langle N_{\mathrm{tr}}\right\rangle$. 
4) A random ensemble with the amplitude coefficient $A_{i}$ of each trap is generated. Simulations are carried out for ensembles with different standard deviations in $A_{i}$, i.e., different values for $\left(\left\langle A^{4}\right\rangle /\left\langle A^{2}\right\rangle^{2}\right)^{0.5}$.

5) The corner frequency $f_{i}$ of each trap in a given sample is chosen by lot according to a uniform distribution for the 268 frequency indexes. Since the corresponding frequency values are distributed in a log scale, they will be distributed according to [2, eq. (27)].

6) To allow the evaluation of the average values and the standard deviations, an ensemble of 620 samples (MOSFETs) is simulated for each pair $\left\langle N_{\mathrm{tr}}\right\rangle$ and $\left(\left\langle A^{4}\right\rangle /\left\langle A^{2}\right\rangle^{2}\right)^{0.5}$.

7) $S(f)$ corresponding to each sample is calculated after (1).

8) $\langle S(f)\rangle^{2}$ and $\left\langle S(f)^{2}\right\rangle$ are evaluated.

9) $\sigma_{(S(f))}$ is then evaluated.

10) Next, the noise power $n p_{\mathrm{BW}}$ and the squared noise power $n p_{\mathrm{BW}}^{2}$ in each of the 620 samples are numerically integrated over 11 different circuit bandwidths, i.e., for 11 different pairs $f_{L}$ and $f_{H}$, after (7) and [2, eq. (63)], respectively.

11) Finally, the mean values $\left\langle n p_{\mathrm{BW}}\right\rangle$ and $\left\langle n p_{\mathrm{BW}}^{2}\right\rangle$, as well as the standard deviation $\sigma_{\mathrm{np}}$, are evaluated for the 11 different circuit bandwidths.

In order to verify the correctness of the derived analytical model equations, the dependence of $\langle S(f)\rangle, \sigma_{S(f)},\left\langle n p_{\mathrm{BW}}\right\rangle$, and $\sigma_{\mathrm{np}}$ on the parameters $N_{\mathrm{dec}}, W, L, A$, and $f$ was compared to the Monte Carlo simulation results. A very good agreement between Monte Carlo simulations and the analytical model was found. To keep the paper compact, only the most relevant results are explicitly shown here, together with experimental data. The bias point dependence of LF noise behavior is discussed in [1] and [2].

For the minimum-size transistors operated in saturation, the experimental value for $\sigma_{S(f)} /\langle S(f)\rangle$ at frequency $f=4 \mathrm{~Hz}$ is 5.4 for the $0.13-\mu \mathrm{m}$ technology node and 5.1 for the $0.09-\mu \mathrm{m}$ technology node. The standard deviation of measurement accuracy is 0.3 and 0.45 , respectively. The experimental values for $\sigma_{\mathrm{np}} /\langle n p\rangle$ at $f_{H} / f_{L}=10^{4} \mathrm{~Hz} / 1 \mathrm{~Hz}$ are $1.9,3.6$, and 3.5 for the minimum-size transistors of the 0.25-, 0.13-, and $0.09-\mu \mathrm{m}$ technology nodes, respectively. The respective values for the standard deviation of measurement accuracy are $0.3,0.3$, and 0.45 . The factor $(2 / \pi) \cdot\left(\left\langle A^{4}\right\rangle /\left\langle A^{2}\right\rangle^{2}\right)^{0.5}$ is then evaluated to be equal to $4.02,4.08$, and 3.30 for the $0.25-, 0.13-$, and $0.09-\mu \mathrm{m}$ technology nodes, respectively. Please note that a normalized standard deviation greater than one, as obtained for the minimum-size devices, does not mean that one can get negative noise power, as discussed in [2].

Fig. 2 shows analytical model results, Monte Carlo simulation results, and experimental data for the normalized standard deviation of the noise power $\sigma_{S(f)} /\langle S(f)\rangle$ as a function of frequency $f$ for minimum-size transistors from the $0.13-\mu \mathrm{m}$ technology node biased in saturation at strong inversion with drain voltage equal to $0.85 \mathrm{~V}$ and gate voltage equal to $0.85 \mathrm{~V}$. Monte Carlo simulation is performed according to the aforementioned algorithm for $\left\langle N_{\mathrm{tr}}\right\rangle=2.7$ (i.e., for $\left.\left(N_{\mathrm{dec}} W L\right)^{-0.5}=1.85\right)$ and $(2 / \pi) \cdot\left(\left\langle A^{4}\right\rangle /\left\langle A^{2}\right\rangle^{2}\right)^{0.5}=4.08$. The new experimental data and Monte Carlo simulation results show that the normalized

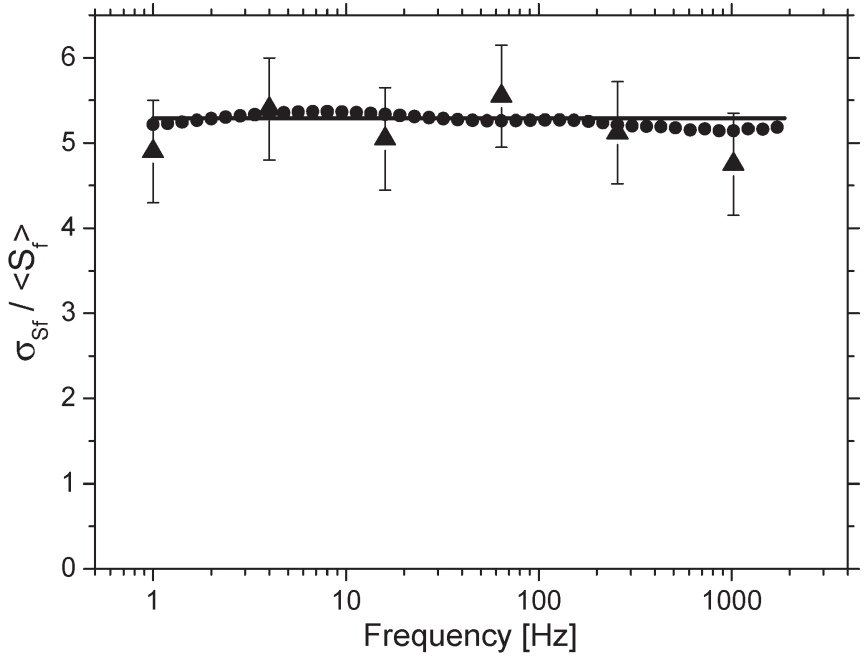

Fig. 2. Normalized standard deviation of the noise power $\left(\sigma_{S(f)} /\langle S(f)\rangle\right)$ as a function of frequency $f$ for minimum-size transistors from the $0.13-\mu \mathrm{m}$ technology node $(W=0.16 \mu \mathrm{m} / L=0.13 \mu \mathrm{m})$. The triangles are the measurement results for transistors biased in saturation. The error bars are the $2 \sigma$ values of measurement accuracy. The circles are the Monte Carlo simulation results. The solid line corresponds to the analytical results, as predicted by (5).

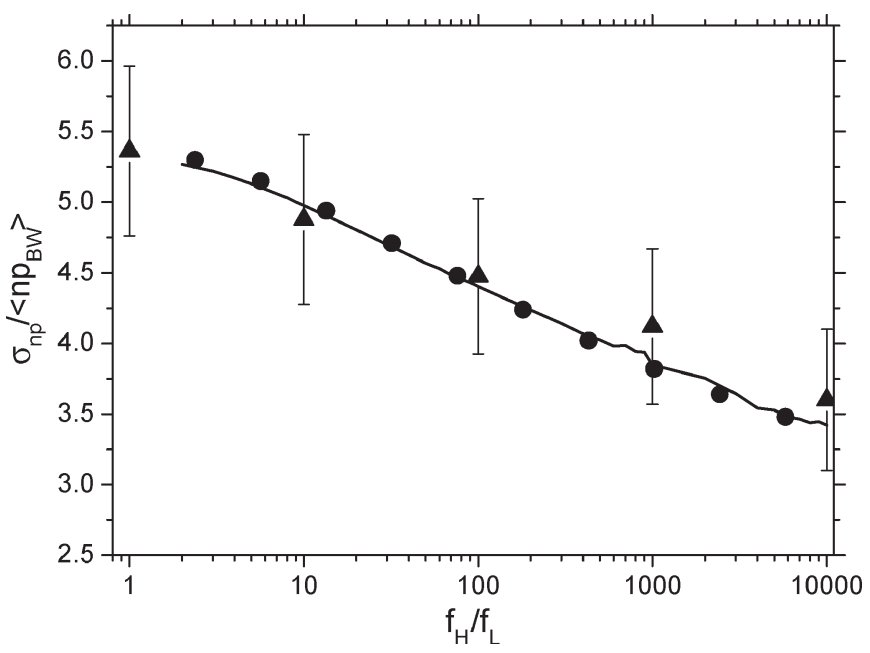

Fig. 3. Dependence of the normalized standard deviation of integrated noise power $\left(\sigma_{\mathrm{np}} /\left\langle n p_{\mathrm{BW}}\right\rangle\right)$ on bandwidth for $f_{L}=1 \mathrm{~Hz}$. The full line shows results from (9), which were obtained by numerical integration of the integral with no analytical solution; the circles are the Monte Carlo simulation results; the triangles show the experimental results for the transistors from the $0.13-\mu \mathrm{m}$ technology node $(W=0.16 \mu \mathrm{m} / L=0.13 \mu \mathrm{m})$ operated in saturation; the error bars are $2 \sigma$ values of measurement accuracy.

standard deviation of the noise power $\sigma_{S(f)} /\langle S(f)\rangle$ is the same at all frequencies $f$. This behavior, predicted by the model presented in [2], is now confirmed by experimental data for different frequencies $f$ and Monte Carlo simulations. Despite limited measurement accuracy, the analytical model, Monte Carlo simulation, and experiment show good agreement.

Fig. 3 shows model results, Monte Carlo simulation results, and experimental data for the dependence of the normalized standard deviation of noise power integrated over the circuit bandwidth $\sigma_{\mathrm{np}} /\left\langle n p_{\mathrm{BW}}\right\rangle$ on circuit bandwidth. The triangles are the experimental results for minimum-size transistors from the $0.13-\mu \mathrm{m}$ technology node operated in saturation at strong 


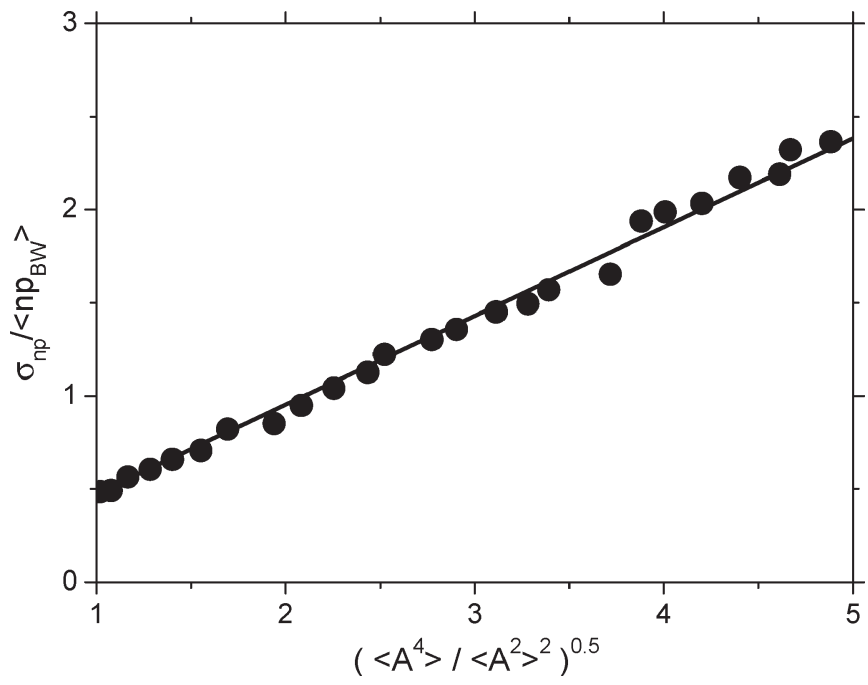

Fig. 4. Dependence of the normalized standard deviation of integrated noise power $\left(\sigma_{\mathrm{np}} /\left\langle n p_{\mathrm{BW}}\right\rangle\right)$ on variability in the amplitude coefficient $A_{i}$ of the Lorentzian corresponding to a trap. The full line shows results from (10); the circles are the Monte Carlo simulation results according to the algorithm presented in Section III. The analytical model and Monte Carlo simulation show good agreement.

inversion with drain voltage equal to $0.85 \mathrm{~V}$ and gate voltage equal to $0.85 \mathrm{~V}$. In the limit $f_{H}=f_{L}$, (5) and (9) become the same, since the last square root in (9) becomes $2^{-0.5}$. Hence, the value shown in Fig. 3 for $f_{H} / f_{L}=1$ is the experimental data for $\sigma_{S(f)} /\langle S(f)\rangle$. The circles are Monte Carlo simulation results for $\left\langle N_{\mathrm{tr}}\right\rangle=2.7$ (i.e., for $\left(N_{\mathrm{dec}} W L\right)^{-0.5}=1.85$ ) and $(2 / \pi) \cdot\left(\left\langle A^{4}\right\rangle /\left\langle A^{2}\right\rangle^{2}\right)^{0.5}=4.08$. The full line is the result, as predicted by (9). In order to evaluate (9), the integral in the square root of this equation was numerically calculated (see Fig. 1). A good agreement between experimental data, numerical simulation, and model (9) is found.

Finally, Fig. 4 describes in detail the noise behavior dependence on the amplitude coefficients $A_{i}$ of trap contribution. As described earlier, a random ensemble with the amplitude coefficients $A_{i}$ of the different traps is generated. Different distributions with different standard deviations were assumed for generating the ensembles of random variables $A_{i}$; for instance, normal, log-normal, Poisson, and uniformly distributed. This allows studying the dependence on $A_{i}$ independent of hypothesis about the actual distribution of the amplitude coefficients $A_{i}$. After the random ensembles for $A_{i}$ are generated, the corresponding values of $\left(\left\langle A^{4}\right\rangle /\left\langle A^{2}\right\rangle^{2}\right)^{0.5}$ are calculated. Results in Fig. 4 are for $N_{\mathrm{dec}} W L=0.6237$ and $f_{H} / f_{L}=10^{4} \mathrm{~Hz} / 1 \mathrm{~Hz}$. The Monte Carlo simulations confirm that $\sigma_{\mathrm{np}} /\left\langle n p_{\mathrm{BW}}\right\rangle$ depends on the variability in amplitude coefficients $A_{i}$ of trap contribution after $\left(\left\langle A^{4}\right\rangle /\left\langle A^{2}\right\rangle^{2}\right)^{0.5}$.

Please note that in all the Monte Carlo simulations run, $A_{i}, f_{i}$, and $N_{\mathrm{tr}}$ are random variables not identically distributed. In model derivation and Monte Carlo simulations, $N_{\text {tr }}$ is assumed to be Poisson distributed, $f_{i}$ is uniformly distributed on a logarithmic scale, and no hypothesis is made about the actual distribution of $A_{i}$ [2], [11]. As an example of possible distributions for $A_{i}$, Fig. 5 shows a numerically generated lognormal distribution of an ensemble of 1000 values for $A_{i}$ that leads to $(2 / \pi) \cdot\left(\left\langle A^{4}\right\rangle /\left\langle A^{2}\right\rangle^{2}\right)^{0.5}=4$. It is important to notice

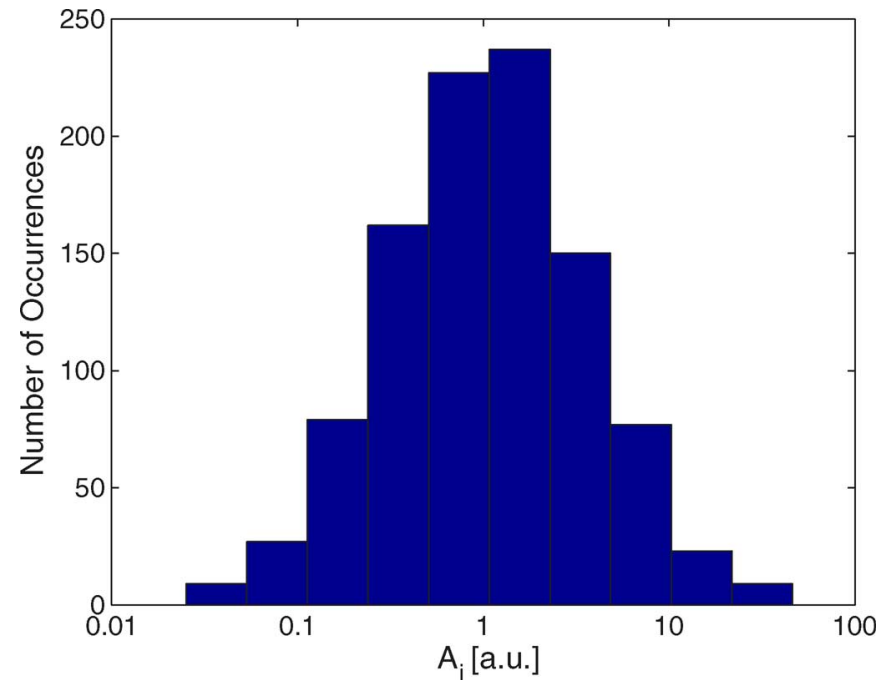

Fig. 5. Histogram of a numerically generated log-normal distribution for $1000 A_{i}$ values as an example of a distribution leading to $(2 / \pi)$. $\left(\left\langle A^{4}\right\rangle /\left\langle A^{2}\right\rangle^{2}\right)^{0.5}=4$.

that $A_{i}$ is not the time-domain RTS amplitude but the amplitude coefficient of the Lorentzian power spectrum.

\section{CONCLUSION}

This paper presents a model for the statistical behavior of LF noise performance of CMOS devices in modern technologies. The developed modeling approach includes the modeling of statistically relevant effects based on microscopically discrete quantities. The model is derived from device physics and is therefore scalable with MOSFET scaling. The analytical formulation is validated through comparison to numerical simulation and experimental results, including the analysis of frequency dependence. For the first time, a formulation for the standard deviation of noise power as a function of circuit bandwidth is provided.

\section{REFERENCES}

[1] R. Brederlow, W. Weber, D. Schmitt-Landsidel, and R. Thewes, "Fluctuations of the low frequency noise of MOS transistors and their modeling in analog and RF-circuits," in IEDM Tech. Dig., 1999, pp. $159-162$.

[2] G. I. Wirth, J. Koh, R. da Silva, R. Thewes, and R. Brederlow, "Modeling of statistical low-frequency noise of deep-submicron MOSFETs," IEEE Trans. Electron Devices, vol. 52, no. 7, pp. 1576-1588, Jul. 2005.

[3] T. Boutchacha and G. Ghibaudo, "Low frequency noise characterization of $0.18 \mu \mathrm{m}$ Si CMOS transistors," Phys. Status Solidi, A, vol. 167, no. 1, pp. 261-270, May 1998.

[4] M. J. Deen, O. Marinov, D. Onsongo, and S. Banerjee, "Low-frequency noise in SiGeC-based pMOSFETs," in Proc. SPIE: Noise Devices and Circuits, 2004, vol. 5470, pp. 215-225.

[5] R. Jayaraman and C. G. Sodini, "A $1 / f$ noise technique to extract the oxide trap density near the conduction band edge of silicon," IEEE Trans. Electron Devices, vol. 36, no. 9, pp. 1773-1782, Sep. 1989.

[6] K. K. Hung, P. K. Ko, C. Hu, and Y. C. Cheng, "A unified model for the flicker noise in metal-oxide-semiconductor field-effect transistors," IEEE Trans. Electron Devices, vol. 37, no. 3, pp. 654-665, Mar. 1990.

[7] K. S. Ralls, W. J. Skocpol, L. D. Jackel, R. E. Howard, A. Fetter, R. W. Epworth, and D. M. Tennat, "Discrete resistance switching in submicrometer silicon inversion layers: Individual interface traps and lowfrequency $(1 / f)$ noise," Phys. Rev. Lett., vol. 52, no. 3, pp. 228-231, Jan. 1984. 
[8] M. Sánden, O. Marinov, M. J. Deen, and M. Ostling, "A new model for the low-frequency noise and the noise level variation in polysilicon emitter BJTs," IEEE Trans. Electron Devices, vol. 49, no. 3, pp. 514-519, Mar. 2002.

[9] E. Simoen, B. Dierickx, C. L. Clayes, and G. J. Declerck, "Explaining the amplitude of RTS noise in submicrometer MOSFETs," IEEE Trans. Electron Devices, vol. 39, no. 2, pp. 419-422, Feb. 1992.

[10] M. J. Kirton and M. J. Uren, "Noise in solid-state microstructures: A new perspective on individual defects, interface states and low-frequency (1/f) noise," Adv. Phys., vol. 38, no. 4, pp. 367-468, Nov. 1989.

[11] R. da Silva, G. I. Wirth, and R. Brederlow, "Novel analytical and numerical approach to modeling low-frequency noise in semiconductor devices," Physica A, Statistical Mech. Appl., vol. 362, no. 2, pp. 277-288, Apr. 2006.

[12] S. Machlup, "Noise in semiconductors: Spectrum of a two-parameter random signal," J. Appl. Phys., vol. 25, no. 3, pp. 341-343, Mar. 1954.

[13] R. Brederlow, W. Weber, R. Jurk, C. Dahl, S. Kessel, J. Holz, W. Sauert, P. Klein, B. Lemaitre, D. Schmitt-Landsiedel, and R. Thewes, "Influence of fluorinated gate oxides on the low frequency noise of MOS transistors under analog operation," in Proc. 28th ESSDERC, 1998, pp. 472-475.

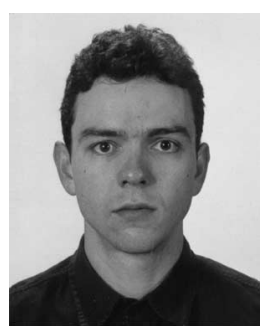

Gilson I. Wirth (S'97-M'99) received the B.S.E.E. and M.Sc. degrees from the Federal University of Rio Grande do Sul, Porto Alegre, Brazil, in 1990 and 1994, respectively, and the Dr.Ing. degree in electrical engineering from the University of Dortmund, Dortmund, Germany, in 1999.

From 2000 to 2002, he was with the Instituto de Informática, Federal University of Rio Grande do Sul, as a Lecturer and Researcher in the field of microelectronics. In 2002, he joined the Department of Computer Engineering, State University of Rio Grande do Sul, where he is currently a Professor and the Head of the Research Group in micro- and nanoelectronics. In July, August, and December 2001, he was with Motorola, Austin, TX, working on CMOS process technology transfer to CEITEC, Porto Alegre, Brazil. In February and March 2002, he was with the Corporate Research Department, Infineon Technologies, Munich, Germany, as a Guest Researcher on low-frequency noise in deep-submicrometer MOS devices. His research interests include low-frequency noise, variability, and design for yield of digital, analog, and mixed-signal circuits.

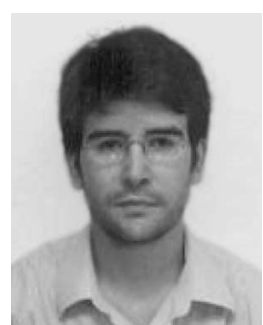

Roberto da Silva was born in Mauá, São Paulo, Brazil, in 1973. He received the B.S. and Ph.D. degrees in physics from the University of São Paulo, in 1997 and 2002, respectively.

From 1997 to 2002, he worked in many areas of statistical mechanics, mathematical physics, and computational physics, where most of his papers are published in indexed journals. Since 2003, he has been with the Federal University of Rio Grande do Sul, Porto Alegre, Brazil, as a Professor. His research interests include numerical and statistical modeling in scientific computing.

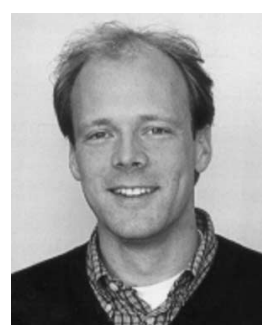

Ralf Brederlow (SM'06) was born in Munich, Germany, in 1970. He received the Dipl.Phys. degree from the Technical University of Munich, Munich, Germany, in 1996, and the Dr.Ing. degree from the Technical University of Berlin, Berlin, Germany, in 1999.

In 1995, he was with the Walter-Schottky Institute, Garching, Germany, working on FIR detectors and $\mathrm{Si} / \mathrm{SiGe}$ MBE. In 1996, he was associated with Siemens Corporate Research, working on the characterization, modeling, and reliability of noise in analog circuits. In 1999, he joined the Corporate Research Department, Infineon Technologies, Munich, as a Project Manager for technology-related circuit design. His work included noise-tolerant analog and digital circuit design, design/technology cooptimization, polymer electronics for RFID, and sensor systems for biochemical applications. In 2005, he joined Infineon's Smart Card Division, managing different analog IP projects for contact-based and air-interface smart cards. Since October 2006, he has been with Texas Instruments Deutschland $\mathrm{GmbH}$, Freising, Germany, leading an analog module design team with focus on low-power analog interfaces for microcontrollers. $\mathrm{He}$ has authored or coauthored some 50 technical publications and is the holder several patents.

Dr. Brederlow is a member of the German Association of Electrical Engineers (VDE) and the German Physical Society (DPG). He is the Technical Program Vice Chair of the 2006 IEDM and a member of the ESSDERC/ESSCIRC Steering Committee. 